\title{
Monitoring and Research on the Aquatic Microbes
}

\author{
Zhang Chunyan \\ Experimentalist \\ School of Environmental Sciences \& Institute of Environmental Biotechnology, Changsha \\ Environmental Protection College
}

Abstract-With the improvement of the overall levels of social development in China as well as the rapid pace of global economic integration, people have higher requirements for improving the living environment. Vast water resources, such as rivers, lakes and seas etc, are important links to improve people's living environment. Excellent water resources have active significance for people's traveling, relaxing themselves as well as improving the local climate. However, as for parts of the water in China, the side effect produced by aquatic microbes in the water often create vicious circles for the water quality and affect people's health and hinder the rapid development of social economy. In this paper, the monitoring of the aquatic microbes will be analyzed and researched to some extent.

Key words- aquatic microbes; monitoring; research

\section{Introduction}

In recent years, with the increase of people's living standard in China, people are yearning for the beautiful life of green hills and clean waters as well as the natural ecology in the rapid development of urban life. Therefore, pursue for the natural beauty and the ecological beauty has been the necessity of the whole society. It is undeniable that excellent aquatic system plays an important part for improving our living standard. The rivers in the city and nearby can effectively provide a good and pleasant living environment for people, which can make them fully taste the beauty of nature and life. However, with the development of industry, it is inevitable that much waste water of industry and life are flowing into aquatic system, which will produce side effect to the water quality because of the large number of microbes in the aquatic system. Therefore, the microbes in the aquatic system should be comprehensively monitored so as to help us better improve the water quality thereby.

\section{PCR Technology}

PCR technology abbreviated from Polymerase Chain Reaction is a technology created in 1980s. PCR is to compound peculiar DNA through enzyme in vitro, which is a method to intercept special DNA fragment by polymerase chain reaction. From the denaturation in high temperature and annealing in low temperature then the extension in temperate temperature, it can form a cycle period during the reaction process so as to rapidly proliferate the target DNA. Due to its strong peculiarity and high sensitivity, it is not only easy to operate but also saves time and energy. Since it has such unique advantages, it is widely applied in the basic research of gene isolation, clone and nucleic acid sequence analysis. With the improvement and study of PCR in recent years by experts from every country, this technology is greatly improved and perfected in this 
process and is widely applied in the environmental microbiology. This technology is to proliferate in vitro by particular gene districts of certain microbes, which can better ascertain the content and category of the microbes in the aquatic system. This method not only has high sensitivity, repeatability as well as good specificity, but also can better supplement the shortcomings in tradition technology which cannot comprehensively reflect the features and diversities of microbes. Meanwhile, for many technologies in China, they identify the DNA sequence of low content in the microbes group based on the proliferation of PCR, which can make an overall analysis to the community structure to the environmental microbes and make this technology better research outlook.

\section{Application of PCR Technology in the Detection of Pathogen in Aquatic Environment}

As for the microbes in the waters, they can be divided into the following four categories, namely bacteria, virus, pathogenic worms as well as protozoans etc, among which many microbes belong to enterogenous, namely they flow into the waters through the feces of humans or animals and intrude the new hosts by new digestive tract after absorbed by animals. Since these waters are most easily to be polluted by the feces of humans and animals and part non-enterogenous bacteria, such as mycobacterium and legionnella are most likely to pollute the waters, it makes the raw water and the microbes existing in the polluted water a threatening healthy problems to people's daily life. In order to minimize the influence caused by such problem, the water quality should be monitored and the handling effect should be actively evaluated. As for the previous monitoring methods for waters, they mainly observe the content and categories in the waters by cultivation pathogens and supervision. However, these methods could not only waste much money and labors but also the accuracy of the aquatic microbes would not be satisfactory. By application of PCR, it can analyze without cultivation and can detect the pathogens more flexible, easy and accurate as well.

In waters, the most dangerous pathogen is virus, which are not only most likely to infect but also requires low dose to cause disease and people often cannot accurately monitoring them. The traditional method is usually to accumulate culture the virus in the waters and detect the virus by electron microscope and other methods. However, the accuracy of this method is low. By PCR technology, however, it can overcome the insufficiency existing in traditional method and improve the quantity and scope of the detection of virus to a large extent. Up to now, scientists all over the world have already successfully detected the HAV and Norwalk virus and can accurately detect the enterovirus and HAV based on the proliferation of cell culture. As for the detective efficiency, this method can improve at least 50\% compared with the single cell culture. It can be said that the application of PCR is an effective method for detecting the aquatic virus.

As for the bacteria contained in the waters, the traditional method is to identify the numbers and categories by isolated culture. However, during the actual application of this method, the selection of culture medium and the methods for isolation can greatly affect the actual detection efficiency. Meanwhile, since parts bacteria cannot grow by artificial medium, it makes the actual detected bacteria far more lower that the actual ones in the aquatic environment not only in numbers but also in 
categories. By application of PCR, it can effectively detect the bacteria in the water, which can greatly improve the detection scope apart from greatly reducing the detection time. In the global research field, there are scientists who have detected the Escherichia Coil cells by PCR proliferation to the 16SrRNA fragment of Escherichia Coil and studied the Escherichia Coil in potable water by PCR through designing the primer to the Eschericia Coli. This method can effectively and flexibly detect the Escherichia Coil in the potable water disinfected by chemical solvent or ozone. The detected result is not only in conformity with the results of traditional filter film cultivation method but also can greatly save our detection time. Besides, the PCR technology uses an special DNA proliferation method, which can accurate detect the bacteria under dormant state and can avoid the lower statistics compared with actual bacteria numbers.

Pathogen is also an important part for the safety of water quality which requires our attention.

But PCR, the DNA template in the water quality sample can realize the detection, which can greatly improve the accuracy and detection efficiency of actual detection. During the application process of this method, it does not require to transfer the indicative microbes but to detect the water samples directly.

\section{Monitoring and Evaluation of PCR Microbes processing procedures}

The current water processing in China can be regarded as the results by studying the reactions of different bacteria. By studying the categories and the species numbers and further obtaining the numbers in the polluted waters, it is of great importance to the stability of different groups in the waters. As is known to all, many pathogenic microbes are grown in polluted water, such as salmonella, Shigella, coxsackie virus as well as hepatitis virus, which can greatly harm people's health. After processing the polluted water, the number of bacteria and virus are greatly reduced and the PCR microbes processing can make great guarantee for people's health.

Meanwhile, it can help us understand that parts of functional bacteria can play a part in degradation of harmful and toxic substance, which is of great active significance to the improvement and upgrading of the future water quality processing technology. At present, molecular biological technique which is based on PCR technology can make comparatively accurate analysis and identification by the difference and different conservatism of the inheritance information of different microbes in the water.

By combing PCR and DEEG technology, it can help us better research the microbes categories of the microbes categories on the biofilm in the rivers and by a series of sequencing and grouping, it can provide vital proof to the current monitoring of the ecological environment of the rivers. By relevant experimental operation. It can better identify the results of the final experiments.

Besides, by PCR technology, the microbes in the sludge of the waters can be analyzed and distinguished. Meanwhile, it is an important way to help us understand the types and structures of the group in the sludge of the waters. By the study of the paper mill, industries and companies, food production factory as well as domestic sewage and so on which have greater influence to the water quality, it can help us master the microbes structures and groups in different types of polluted waters, which is of great positive significance to the monitoring of microbes groups and the processing methods of waste water. 


\section{Conclusions}

In a word, under current living environment, guarantee of the safety and cleanness of the water resources is of crucial importance to our living environment and our health, which requires our sufficient attention. In the previous part, analysis and research have been carried on about the relevant technology and the application scope for the monitoring of the aquatic microbes. In the concrete operation process, theory should be fully connected with the practice, which can better monitor the aquatic microbes more targetedly.

\section{References:}

[1]Wu Shenshu,Tan Meiying \& Liu Wei.Control Effect of the Methane Project on the Costs of Polluted Environment by Animal Feces [J]. Chinese Journal of Eco-Agriculture.2012(02).
[2]Liu Xinwei \& Lv Xiaojie. Discussion on the Application of the Standard Plate Count on the Aquatic Environment

$[\mathrm{J}]$.

Environmental Protection and

Circular Economy.2011(10):46-47.

[3] Xie Weiwei, Zhou Guangzhu, Guo Yanxu \& Li Huai.Study on the Scenery Reconstruction and Ecological Restoration of Vegetational Types of Chaoyang of Daling River[J].Modern Agriculture Science and Technology.2008(04):198-200.

[4] Wang Dongmei.Status Quo and Countermeasures for the Water Pollution of Daling River (Jinshou period)[J]. Heilongjiang Environmental Journal.2011(01):12-13.

[5] Sun Song. Application of Seasonal Kendall Testing Method in the Analysis of the Tendency of Water Quality in Huangtan Reservoir [J]. Environmental Study and Monitoring.2011(02): 53-55. 